\title{
Exploitation of Heterobeltiosis in Tomato (Solanum lycopersicum L.)
}

\author{
Mayur Kumar Sonagara ${ }^{\text {* }}$, Jyotindra N. Patel $^{2}$, R. R. Acharya ${ }^{3}$ and \\ Deepak D. Sharma ${ }^{1}$ \\ ${ }^{1}$ Department of Genetics and Plant Breeding, B. A. College of Agriculture, \\ Anand Agricultural University, Anand, Gujarat, India \\ ${ }^{2}$ Bidi Tobacco Research Station, Anand Agricultural University, Anand, Gujarat, India \\ ${ }^{3}$ Main Vegetable Research Station, Anand Agricultural University, Anand, Gujarat, India \\ *Corresponding author
}

\section{A B S T R A C T}

\begin{tabular}{l} 
Key w o r d s \\
Tomato, \\
Heterobeltiosis, \\
Hybrids, \\
Diverse, Diallel \\
\hline Article Info \\
\hline $\begin{array}{l}\text { Accepted: } \\
\text { 07 March } 2020 \\
\text { Available Online: } \\
10 \text { April } 2020\end{array}$ \\
\hline
\end{tabular}

Heterobeltiosis analysis was undertaken using ten diverse lines and their 45 $\mathrm{F}_{1}$ hybrids of tomato developed through diallel fashion excluding reciprocals. The experimental material was evaluated in a randomized complete block design with three replications at the Main Vegetable Research Station, Anand Agricultural University, Anand, during kharifrabi, 2018-19.Among 45 hybrids tested, 14 exhibited significant positive heterobeltiosis for fruit yield per plant. Out of these the best three heterotic hybrids in order were ATL-17-05 × 2016/TODVAR-3 (202.80\%), GAT-5 $\times$ 2014/TOLCVRES-1 (178.21\%) and GAT-5 × DVRT-2 (132.76\%).

\section{Introduction}

Tomato (Solanum lycopersicum L.) is an important and one of the most widely grown vegetable crops of both tropics and sub tropics of the world (Kalloo, 1988). Tomato ranks third in priority after potato and onion in India but ranks second after potato in the world. India ranks second in the area (814 thousand hectares) and production (20515 thousand metric tons) in the world (Anonymous 2018). This crop exhibits rich genetic diversity for various horticultural traits and has a scope for its improvement. In tomato, the comparative ease of emasculation, high percentage of fruit setting and good number of seeds per fruit also facilitate the exploitation of heterosis.

The major objective in the most tomato breeding programmes is to improve the genetic potential for fruit yield and quality. Yield being a complex quantitative character in tomato can be improved through improving 
its contributing traits, i.e. mean fruit weight, number of fruits per plant, fruit length, and breadth and plant vigour. Heterosis for various fruit quantitative and quality characters has been reported by Rajan(2014) and Panchal et al., (2017) in tomato. An added advantage of the diallel analysis is that it provides an overall genetic picture of experimental material in a single generation. Hence, present investigation was carried out at Main Vegetable Research Station, Anand Agricultural University, Anand, Gujarat during kharif-rabi, 2018-19to assess the heterosis levels expressed by hybrids over their better parents for Yields and yield attributing traits taken under consideration and also to identify best heterotic combinations for the same.

\section{Materials and Methods}

The experimental material comprised of 10 diverse lines viz., ATL-17-02 ATL-17-06 ATL-17-05, ATL-16-07, GAT-5, DVRT-2, 2014/TOLCVRES-1, 2016/TODVAR-2, 2016/TODVAR-3 and 2014/TOLCVRES-3 of tomato. The crosses were made in diallel fashion excluding reciprocals, during kharifrabi 2017-18. The $45 \mathrm{~F}_{1}$ hybrids along with their 10 parents were evaluated in a randomized complete block design with three replications at the Main Vegetable Research Station, Anand Agricultural University, Anand, during kharif-rabi 2018-19.

Each experimental unit was represented by a single row of $3.6 \mathrm{~m}$ length accommodating eight plants with inter and intra row spacing of $90 \mathrm{~cm}$ and $45 \mathrm{~cm}$, respectively. The recommended cultural practices and plant protection measures obligatory to raise healthy crop were followed. Observations were recorded on randomly selected five plants in each experimental unit for eight maturity characters viz., days to initiation of flowering, plant height $(\mathrm{cm})$, days to first fruit ripening, average fruit weight, fruit yield per plant, 1000 seed weight (g), number of fruits per plant, and number of locules per fruit.

\section{Statistical analysis}

\section{Heterobeltiosis (HB):}

The estimation of heterosis over better parent and over standard check is more realistic. Hence, in present investigation, heterosis was estimated over better parent (BP) referred to as heterobeltiosis (HB).

Heterosis expressed in percentage will be calculated as the deviation of $F_{1}$ over better parent (Fonseca and Patterson, 1968) by following formulae:

$$
\text { Heterbeltosis }(\%)=\frac{\overline{F_{1}}-\overline{B P}}{\overline{B P}} \times 100
$$

Where,

$\overline{F_{1}}=$ Mean performance of $F_{1}$

$\overline{B P}=$ Mean value of better parent of respective cross combination

\section{Results and Discussion}

The study of heterobeltiosis provide only the percent increase of $F_{1}$ over the better parent, thus helps in identifying the best crosses but fails to identify the possible causes for superiority of hybrids. It is an effective tool in improving the yield and component traits of different crop species. The heterosis breeding has been extensively utilized in improving yield particularly in tomato crop. The heterobeltiosis for seed yield and its components in linseed are presented in Table 1.

\section{Days to initiation of flowering}

For this trait, negative heterobeltiosis is of main interest to the breeder because it is always desirable to incorporate earliness, 
hence more attention was given towards negative heterobeltiosis. The heterobeltiosis of this trait ranged from $-18.02 \%$ (ATL-17-05 $\times$ 2016/TODVAR-3) to $31.52 \%$ (2014/ TOLCVRES-1 $\times$ 2016/TODVAR-3). One hybrid out of forty five hybrid viz., ATL-17$02 \times$ ATL-16-07 (-13.73\%) registered significant and negative heterobeltiosis.

The results are in agreement with the findings of Baishya et al., (2001), Joshi and Thakur (2003), Duhan et al., (2005), Singh (2005), Hannan et al., (2007a), Ahmad et al., (2015) and Chauhan et al., (2014) for heterobeltiosis in positive as well as in negative direction.

\section{Plant height}

The positive heterosis is desirable for this character. The heterobeltiosis varied from $48.21 \%$ (ATL-17-06 × 2016/TODVAR-3) to $62.16 \%$ (2016/TODVAR-2 × 2014/ TOLCVRES-3). Out of 45 hybrids, total 6 hybrids had significant positive heterobeltiosis for this trait. The best three hybrids 2016/ TODVAR-2 × 2014/ TOLCVRES-3 (62.16\%), ATL-17-02 × 2016 /TODVAR-2 (36.92\%) and GAT-5 × 2016/ TODVAR-2 (36.13\%), had registered significant heterosis in desirable direction over respective better parent.

For plant height, the results are in agreement with the findings of Tiwari and Lal (2004), Duhan et al., (2005), Singh (2005), Ahmad et al., (2015), Kumari and Sharma (2011), Yadav et al., (2013) and Bhalala (2018)for heterobeltiosis in negative as well as in positive direction.

\section{Days to first fruit ripening}

For earliness, heterotic effects in negative direction are desirable. The heterobeltiosis ranged from $-18.60 \%$ (2016/TODVAR-2 $\times$ 2014/TOLCVRES-3) to $48.79 \%$ (ATL-17-06 $\times$ 2016/ TODVAR-3). One hybrid viz., 2016/TODVAR-2 × 2014/TOLCVRES-3 ($18.60 \%$ ) documented significant and negative heterobeltiosis.

The results are in conformity with the reports of Singh (2005), Hannan et al., (2007a), and Bhalala (2018) for heterobeltiosis in negative direction as well as in positive directions.

\section{Average fruit weight}

The positive heterobeltiosis is desirable for this character. The heterobeltiosis was varied from $-62.55 \% \quad$ (ATL-17-05 $\times$ 2014/TOLCVRES-3) to $45.16 \%$ (ATL-16-07 $\times$ GAT-5). Out of 45 hybrids, 6 had significant positive heterobeltiosis for this trait. The best three hybrids ATL-16-07 $\times$ GAT-5 (45.16\%), ATL-17-02 ×DVRT-2 $(38.78 \%)$ and GAT-5 × 2014/TOLCVRES-3 $(31.91 \%)$, had registered significant heterosis in desirable direction over respective better parent.

The findings are in concordant with the reportsof Singh (2005), Kumari and Sharma (2011), Yadav et al., (2013), Dagade et al., (2015a), Kumar et al., (2016) and Triveni et al., (2017) for heterobeltiosis in negative direction as well as in positive directions.

\section{Number of fruits per plant}

The positive heterobeltiosis is desirable for this character. The heterobeltiosis varied from $-92.29 \%$ (GAT-5 × 2014/TOLCVRES-3) to $237.95 \%$ (GAT-5 × DVRT-2). Out of 45 hybrids, 13 hybrids had shown significant positive heterobeltiosis for this trait. The best three hybrid combinations were GAT-5 $\times$ DVRT-2 (237.95\%), ATL-17-05 $\times$ 2014/TOLCVRES-3 (88.79\%) and DVRT-2 $\times$ 2016/TODVAR-2 (61.18\%).

The results are in agreement with the findings 
of Baishya et al., (2001), Joshi and Thakur (2003) (HB), Singh et al., (2012), Ahmad et al., (2015), Aisyah et al., (2016), Kumar et al., (2016) for heterobeltiosis in negative as well as in positive direction.

\section{Fruit yield per plant}

The positive heterobeltiosis is desirable for this character. The heterobeltiosis was varied from $-91.85 \%$ (ATL-17-05 $\times$ ATL-16-07) to 202.80\% (ATL-17-05 × 2016/TODVAR-3). Out of 45 hybrids, 14 had significant positive heterobeltiosis for this trait. The best three hybrids ATL-17-05 $\times$ 2016/TODVAR-3 (202.80\%), GAT-5 × 2014/TOLCVRES-1 $(178.21 \%)$ and GAT-5 × DVRT-2 (132.76\%), had registered significant heterosis in desirable direction over respective better parent.

The findings are in agreement with the reports of Singh (2005), Hannan et al., (2007a), Kumari and Sharma (2011), Yadav et al., (2013), Kumar et al., (2016), Triveni et al., (2017) and Bhalala (2018) for heterobeltiosis in negative direction as well as in positive directions.

\section{0 seed weight}

1000 seed weight is also one of the important traits and positive heterosis is desirable for this trait. The heterosis over better parent varied from $-32.78 \%$ (ATL-16-07 × DVRT-2) to $33.00 \%$ (ATL-17-02 $\times$ ATL-17-05). Out of 45 hybrids, only 2 hybrids ATL-17-02 $\times$ ATL-17-05 (33.00\%) and ATL-16-07 $\times$ 2014/TOLCVRES-1 (9.38\%) had registered significant positive heterobeltiosis for this trait.

Significant estimates of heterosis for 1000 seed weight in tomato has been reported byPatwary et al., (2013) and Bhalala (2018) which shows heterobeltiosis in negative direction as well as in positive directions.

\section{Pericarp thickness}

The positive heterosis is desirable for this character. The heterobeltiosis was varied from $-34.15 \%$ (ATL-17-06 × 2014/TOLCVRES-1) to $20.29 \%$ (GAT-5 $\times 2014 /$ TOLCVRES-3). Out of 45 hybrids only three hybrids GAT $-5 \times$ 2014/TOLCVRES-3 (20.29\%), ATL-17-02 $\times$ GAT-5 (17.18\%) and ATL-17-06 $\times$ 2016/TODVAR-3 (10.37\%), had taped significant positive heterobeltiosis for this trait.

For pericarp thickness, the results are in agreement with the findings of Kumari and Sharma (2011), Kumar et al., (2013)Rajan (2014), Dagade et al., (2015a) Sharma and Bhardwaj, (2016), Aisyah et al., (2016), Kumar and Paliwal (2016) and Madhvi et al., (2018a) for heterobeltiosis in negative as well as in positive direction.

\section{Number of locules per fruit}

The positive heterosis is desirable for this character. The heterobeltiosis varied from 45.07\% (ATL-17-05 × 2014/TOLCVRES-1) to $78.00 \%$ (2016/TODVAR-2 $\times$ 2014/ TOLCVRES-3). Out of 45 hybrids, 18 had shown significant positive heterobeltiosis for this trait.

The best three hybrid combinations were 2016/TODVAR-2 × 2014/TOLCVRES-3 (78.00\%), 2016/TODVAR-3 $\times$ 2014/TOLCVRES-3 (57.41\%) and ATL-17$02 \times 2014 /$ TOLCVRES-3 (42.22\%).

For number of locules the findings are in concordant to the reports of Singh and Singh (2003), Joshi et al., (2005), Ahmad et al., (2011), Singh et al., (2012), Sharma and Bhardwaj (2014), Savita and Singh (2015) and Aisyah et al., (2016) for heterobeltiosis in negative as well as in positive direction. 
Table.1.1 Estimate of heterobeltiosis (HB) in per cent for yield and its attributing characters

\begin{tabular}{|c|c|c|c|c|c|}
\hline Sr. No. & Hybrids & $\begin{array}{c}\text { Days to } \\
\text { initiation of } \\
\text { flowering }\end{array}$ & $\begin{array}{l}\text { Plant } \\
\text { height }\end{array}$ & $\begin{array}{l}\text { Days to } \\
\text { first fruit } \\
\text { ripening }\end{array}$ & $\begin{array}{c}\text { Average } \\
\text { fruit weight }\end{array}$ \\
\hline & $(1)$ & (2) & (3) & (4) & (5) \\
\hline 1 & ATL-17-02 $\times$ ATL-17-06 & 5.15 & $-40.18 * *$ & $14.11 *$ & $-33.68 * *$ \\
\hline 2 & ATL-17-02 $\times$ ATL-17-05 & -6.86 & -4.88 & 0.00 & $-17.64 *$ \\
\hline 3 & ATL-17-02 × ATL-16-07 & $-13.73^{*}$ & $-20.96 * *$ & -4.04 & -10.81 \\
\hline 4 & ATL-17-02 × GAT-5 & 10.78 & $-23.96 * *$ & 12.50 & $29.86 * *$ \\
\hline 5 & ATL-17-02 × DVRT-2 & 17.39* & $-30.27 * *$ & 7.95 & $38.78 * *$ \\
\hline 6 & $\begin{array}{l}\text { ATL-17-02 } \times \\
\text { 2014/TOLCVRES-1 }\end{array}$ & -7.61 & $-19.45 * *$ & -4.41 & -1.32 \\
\hline 7 & ATL-17-02 × 2016/TODVAR-2 & -9.81 & $36.92 * *$ & -2.94 & $-8.42 * *$ \\
\hline 8 & ATL-17-02 × 2016/TODVAR-3 & 2.94 & $-21.01 *$ & $30.88 * *$ & $4.90 * *$ \\
\hline 9 & $\begin{array}{l}\text { ATL-17-02 } \times \\
\text { 2014/TOLCVRES-3 }\end{array}$ & -4.90 & $-36.49 * *$ & -0.37 & $-42.93 *$ \\
\hline 10 & ATL-17-06 $\times$ ATL-17-05 & 11.34 & $-30.51 * *$ & 14.92 & $26.62 * *$ \\
\hline 11 & ATL-17-06 × ATL-16-07 & $\mathbf{1 7 . 5 3}$ & $-26.43 * *$ & $32.26 * *$ & $-43.31 * *$ \\
\hline 12 & ATL-17-06 × GAT-5 & 23.71* & -38.05 & $25.40 *$ & 0.59 \\
\hline 13 & ATL-17-06 × DVRT-2 & 9.28 & $-17.53 *$ & 8.06 & $-21.85 *$ \\
\hline 14 & $\begin{array}{l}\text { ATL-17-06 } \times \\
\text { 2014/TOLCVRES-1 }\end{array}$ & 0.00 & $-33.46 * *$ & 2.82 & -13.99 \\
\hline 15 & ATL-17-06 $\times$ 2016/TODVAR-2 & -3.09 & $-3.92 * *$ & 12.10 & $-50.29 * *$ \\
\hline 16 & ATL-17-06 × 2016/TODVAR-3 & 7.22 & $-48.21 *$ & 48.79** & $-29.38 * *$ \\
\hline 17 & $\begin{array}{l}\text { ATL-17-06 } \times \\
\text { 2014/TOLCVRES-3 }\end{array}$ & $23.71 *$ & -28.20 & $25.81 *$ & $-30.21 * *$ \\
\hline 18 & ATL-17-05 × ATL-16-07 & $19.23 * *$ & $-29.31 * *$ & $17.98 *$ & $-24.03 *$ \\
\hline 19 & ATL-17-05 × GAT-5 & -5.50 & $-10.35 * *$ & $23.44 * *$ & -10.89 \\
\hline 20 & ATL-17-05 × DVRT-2 & $15.22 *$ & $-3.75 * *$ & -6.82 & -13.23 \\
\hline 21 & $\begin{array}{l}\text { ATL-17-05 } \times \\
\text { 2014/TOLCVRES-1 }\end{array}$ & 8.70 & -2.21 & -2.25 & -7.70 \\
\hline 22 & ATL-17-05 × 2016/TODVAR-2 & 6.12 & $-16.30 *$ & $15.36 *$ & $-34.69 * *$ \\
\hline 23 & ATL-17-05 × 2016/TODVAR-3 & -18.02 & $9.44 * *$ & -6.74 & $-24.91 * *$ \\
\hline 24 & $\begin{array}{l}\text { ATL-17-05 } \times \\
\text { 2014/TOLCVRES-3 }\end{array}$ & 3.60 & $-6.80 * *$ & -3.37 & $-62.55 * *$ \\
\hline 25 & ATL-16-07 × GAT-5 & $28.85 * *$ & $-14.45 *$ & $18.75 * *$ & $45.16 * *$ \\
\hline 26 & ATL-16-07 × DVRT-2 & 14.13 & -40.59 & 7.95 & $-39.62 * *$ \\
\hline 27 & $\begin{array}{l}\text { ATL-16-07 } \times \\
\text { 2014/TOLCVRES-1 }\end{array}$ & 13.04 & -8.97 & 10.26 & 19.24 \\
\hline 28 & ATL-16-07 × 2016/TODVAR-2 & 12.24 & $-46.41 *$ & $6.35 * *$ & $-51.55 * *$ \\
\hline 29. & ATL-16-07 × 2016/TODVAR-3 & 7.69 & -36.60 & $15.00 *$ & -0.01 \\
\hline 30 & ATL-16-07 $x$ & $17.31 * *$ & $-5.39 * *$ & $7.69 * *$ & $-35.03 * *$ \\
\hline
\end{tabular}




\begin{tabular}{|c|c|c|c|c|c|}
\hline & 2014/TOLCVRES-3 & & & & \\
\hline 31 & GAT-5 × DVRT-2 & 1.09 & -0.54 & 2.73 & $22.39 * *$ \\
\hline 32 & GAT-5 $\times$ 2014/TOLCVRES-1 & -5.43 & 7.24 & 3.91 & 9.11 \\
\hline 33 & GAT-5 × 2016/TODVAR-2 & $19.39 * *$ & $36.13 * *$ & 16.41 & $-41.02 * *$ \\
\hline 34 & GAT-5 × 2016/TODVAR-3 & -12.84 & $10.44 * *$ & 1.56 & $-17.69 *$ \\
\hline 35 & GAT-5 $\times$ 2014/TOLCVRES-3 & -9.17 & -9.13 & 15.23 & $31.91 * *$ \\
\hline 36 & DVRT-2 $\times$ 2014/TOLCVRES-1 & 5.43 & $-27.92 *$ & -2.65 & -11.51 \\
\hline 37 & DVRT-2 × 2016/TODVAR-2 & 2.17 & $-7.77 *$ & -3.79 & $-39.17 * *$ \\
\hline 38 & DVRT-2 $\times$ 2016/TODVAR-3 & 8.70 & $6.96 * *$ & 2.27 & -6.98 \\
\hline 39 & DVRT-2 $\times$ 2014/TOLCVRES-3 & 11.96 & -15.03 & 3.03 & -9.16 \\
\hline 40 & $\begin{array}{l}\text { 2014/TOLCVRES-1 } \times \\
\text { 2016/TODVAR-2 }\end{array}$ & -1.09 & $-16.04 * *$ & -4.03 & $-45.00 * *$ \\
\hline 41 & $\begin{array}{l}\text { 2014/TOLCVRES-1 } \times \\
\text { 2016/TODVAR-3 }\end{array}$ & $31.52 * *$ & -36.13 & $16.85^{* *}$ & $-16.03 *$ \\
\hline 42 & $\begin{array}{l}\text { 2014/TOLCVRES-1 } \times \\
\text { 2014/TOLCVRES-3 }\end{array}$ & 9.78 & -33.37 & -6.96 & $-33.75 * *$ \\
\hline 43 & $\begin{array}{l}\text { 2016/TODVAR-2 } \times \\
\text { 2016/TODVAR-3 }\end{array}$ & $20.41 *$ & $29.11 * *$ & 14.64* & -28.96 \\
\hline 44 & $\begin{array}{l}\text { 2016/TODVAR-2 } \times \\
\text { 2014/TOLCVRES-3 }\end{array}$ & 4.08 & $62.16 * *$ & $-18.60 * *$ & $-6.27 * *$ \\
\hline 45 & $\begin{array}{l}\text { 2016/TODVAR-3 } \times \\
\text { 2014/TOLCVRES-3 }\end{array}$ & $5.17 * *$ & -1.25 & 0.00 & -18.54 \\
\hline \multirow[t]{2}{*}{ Range } & Min. & -18.02 & -48.21 & -18.60 & -62.55 \\
\hline & Max. & 31.52 & 62.16 & 48.79 & 45.16 \\
\hline \multirow{3}{*}{$\begin{array}{l}\text { No. of } \\
\text { significant } \\
\text { crosses }\end{array}$} & Positive & 11 & 6 & 15 & 6 \\
\hline & Negative & 1 & 24 & 1 & 23 \\
\hline & Total crosses & 12 & 30 & 16 & 29 \\
\hline S. E. \pm & & 2.38 & 13.92 & 5.6 & 4.61 \\
\hline
\end{tabular}


Table 1.2

\begin{tabular}{|c|c|c|c|c|c|}
\hline Sr. No. & Hybrids & $\begin{array}{c}\text { Number of } \\
\text { fruits per plant }\end{array}$ & $\begin{array}{l}\text { Fruit yield } \\
\text { per plant }\end{array}$ & $\begin{array}{l}1000 \text { seed } \\
\text { weight }\end{array}$ & $\begin{array}{l}\text { Pericarp } \\
\text { thickness }\end{array}$ \\
\hline & (1) & (2) & (3) & (4) & (5) \\
\hline 1 & ATL-17-02 $\times$ ATL-17-06 & $-52.91 * *$ & $-46.85 * *$ & $-13.60 *$ & -0.61 \\
\hline 2 & ATL-17-02 $\times$ ATL-17-05 & $71.39 * *$ & $65.43 * *$ & 33.00** & -7.98 \\
\hline 3 & ATL-17-02 × ATL-16-07 & $45.83 * *$ & $40.06 * *$ & -11.13 & 0.00 \\
\hline 4 & ATL-17-02 × GAT-5 & $-58.77 * *$ & $-50.96 * *$ & $-25.75 * *$ & $17.18 * *$ \\
\hline 5 & ATL-17-02 $\times$ DVRT-2 & $-30.50 * *$ & $-28.47 * *$ & -4.91 & 0.00 \\
\hline 6 & $\begin{array}{l}\text { ATL-17-02 } \times \\
\text { 2014/TOLCVRES-1 }\end{array}$ & $-25.53 * *$ & $-16.78 *$ & -6.13 & -7.98 \\
\hline 7 & $\begin{array}{l}\text { ATL-17-02 } \times \\
\text { 2016/TODVAR-2 }\end{array}$ & -1.71 & 15.70 & -7.36 & -1.84 \\
\hline 8 & $\begin{array}{l}\text { ATL-17-02 } \times \\
\text { 2016/TODVAR-3 }\end{array}$ & -12.86 & $-34.79 * *$ & $-14.60 *$ & $-11.66 *$ \\
\hline 9 & $\begin{array}{l}\text { ATL-17-02 } \times \\
\text { 2014/TOLCVRES-3 }\end{array}$ & -10.08 & -28.18 & $-22.30 * *$ & $-18.40 * *$ \\
\hline 10 & ATL-17-06 × ATL-17-05 & $-65.45 * *$ & $-54.55 * *$ & 8.96 & 0.61 \\
\hline 11 & ATL-17-06 × ATL-16-07 & $78.80 * *$ & $37.01 * *$ & $-31.24 * *$ & $-18.29 * *$ \\
\hline 12 & ATL-17-06 × GAT-5 & $-73.46 * *$ & $-58.53 * *$ & -13.91 & -18.90 \\
\hline 13 & ATL-17-06 × DVRT-2 & $-49.89 * *$ & $-17.82 *$ & $-20.65 * *$ & $-21.34 * *$ \\
\hline 14 & $\begin{array}{l}\text { ATL-17-06 } \times \\
\text { 2014/TOLCVRES-1 }\end{array}$ & 1.97 & 0.00 & $-17.89 * *$ & $-34.15 * *$ \\
\hline 15 & $\begin{array}{l}\text { ATL-17-06 } \times \\
\text { 2016/TODVAR-2 }\end{array}$ & $12.42 *$ & -6.39 & -4.39 & -8.54 \\
\hline 16 & $\begin{array}{l}\text { ATL-17-06 } \times \\
\text { 2016/TODVAR-3 }\end{array}$ & -14.31 & $-66.37 * *$ & 16.42 & $10.37 * *$ \\
\hline 17 & $\begin{array}{l}\text { ATL-17-06 } \times \\
\text { 2014/TOLCVRES-3 }\end{array}$ & $-29.85 * *$ & $-37.59 * *$ & $-19.46 * *$ & -7.93 \\
\hline 18 & ATL-17-05 × ATL-16-07 & $-89.36 * *$ & $-91.85 * *$ & 9.48 & $-15.95 * *$ \\
\hline 19 & ATL-17-05 × GAT-5 & $15.36 *$ & $-28.40 * *$ & $-7.68 * *$ & -1.23 \\
\hline 20 & ATL-17-05 × DVRT-2 & $35.09 * *$ & $38.54 * *$ & 17.29 & $-13.50 *$ \\
\hline 21 & $\begin{array}{l}\text { ATL-17-05 } \times \\
\text { 2014/TOLCVRES-1 }\end{array}$ & $41.85 * *$ & $42.65 * *$ & $-6.15 * *$ & -3.07 \\
\hline 22 & $\begin{array}{l}\text { ATL-17-05 } \times \\
\text { 2016/TODVAR-2 }\end{array}$ & $-64.05 * *$ & $-57.91 * *$ & 4.10 & 4.29 \\
\hline 23 & $\begin{array}{l}\text { ATL-17-05 } \times \\
\text { 2016/TODVAR-3 }\end{array}$ & $77.52 * *$ & $202.80 * *$ & $-2.43 *$ & $-29.45 * *$ \\
\hline 24 & $\begin{array}{l}\text { ATL-17-05 } \times \\
\text { 2014/TOLCVRES-3 }\end{array}$ & $88.79 * *$ & $75.02 * *$ & $-19.58 * *$ & -6.13 \\
\hline 25 & ATL-16-07 × GAT-5 & $-65.52 * *$ & $-46.99 * *$ & $-22.89 *$ & 0.00 \\
\hline
\end{tabular}




\begin{tabular}{|c|c|c|c|c|c|}
\hline 26 & ATL-16-07 × DVRT-2 & -16.31 & -4.86 & $-32.78 * *$ & 20.25 \\
\hline 27 & $\begin{array}{l}\text { ATL-16-07 } \times \\
\text { 2014/TOLCVRES-1 }\end{array}$ & $40.12 * *$ & -4.23 & $9.38 * *$ & -15.63 \\
\hline 28 & $\begin{array}{l}\text { ATL-16-07 } \times \\
\text { 2016/TODVAR-2 }\end{array}$ & $-51.26 * *$ & $-56.25 * *$ & -14.02 & -1.87 \\
\hline 29. & $\begin{array}{l}\text { ATL-16-07 } \times \\
\text { 2016/TODVAR-3 }\end{array}$ & $-53.21 * *$ & $-65.55 * *$ & $-28.56 * *$ & -6.87 \\
\hline 30 & $\begin{array}{l}\text { ATL-16-07 } \times \\
\text { 2014/TOLCVRES-3 }\end{array}$ & -15.42 & 10.82 & -18.76 & -10.00 \\
\hline 31 & GAT-5 × DVRT-2 & $237.95 * *$ & $132.76 * *$ & -4.69 & $-11.66 *$ \\
\hline 32 & GAT-5 $\times$ 2014/TOLCVRES- 1 & -42.85 & $178.21 * *$ & 0.34 & -8.12 \\
\hline 33 & GAT-5 × 2016/TODVAR-2 & 86.70 & -10.90 & -11.68 & -10.74 \\
\hline 34 & GAT-5 $\times 2016 /$ TODVAR-3 & 37.98 & -19.42 & $-30.46 * *$ & $-23.08 * *$ \\
\hline 35 & GAT-5 $\times$ 2014/TOLCVRES-3 & $-92.29 * *$ & -25.45 & -1.06 & $20.29 * *$ \\
\hline 36 & $\begin{array}{l}\text { DVRT-2 } \times \\
\text { 2014/TOLCVRES-1 }\end{array}$ & $-57.28 *$ & 5.40 & 7.14 & $-21.47 * *$ \\
\hline 37 & DVRT-2 × 2016/TODVAR-2 & $61.18^{* *}$ & $44.89 * *$ & 12.95 & -8.59 \\
\hline 38 & DVRT-2 $\times 2016 /$ TODVAR-3 & $-19.79 *$ & $-24.08 * *$ & 6.70 & -9.20 \\
\hline 39 & $\begin{array}{l}\text { DVRT-2 } \times \\
\text { 2014/TOLCVRES-3 }\end{array}$ & 10.79 & $68.50^{* *}$ & -5.80 & -1.84 \\
\hline 40 & $\begin{array}{l}\text { 2014/TOLCVRES-1 } \times \\
\text { 2016/TODVAR-2 }\end{array}$ & $27.88^{* *}$ & $21.00^{* *}$ & $-13.25 *$ & 10.00 \\
\hline 41 & $\begin{array}{l}\text { 2014/TOLCVRES-1 } \times \\
\text { 2016/TODVAR-3 }\end{array}$ & -19.51 & $-28.54 *$ & $-19.82 * *$ & $-12.50 * *$ \\
\hline 42 & $\begin{array}{l}\text { 2014/TOLCVRES-1 } 1 \times \\
\text { 2014/TOLCVRES-3 }\end{array}$ & $-3.21 *$ & 3.84 & -8.61 & -6.25 \\
\hline 43 & $\begin{array}{l}\text { 2016/TODVAR-2 } \times \\
\text { 2016/TODVAR-3 }\end{array}$ & $-28.29 * *$ & $-31.99 * *$ & $-11.79 *$ & 10.07 \\
\hline 44 & $\begin{array}{l}\text { 2016/TODVAR-2 } \times \\
\text { 2014/TOLCVRES-3 }\end{array}$ & 10.67 & $42.40^{* *}$ & 3.58 & 9.40 \\
\hline 45 & $\begin{array}{l}\text { 2016/TODVAR-3 } \times \\
\text { 2014/TOLCVRES-3 }\end{array}$ & 62.14 & $77.01 * *$ & 10.14 & 13.29 \\
\hline Range & Min. & -92.29 & -91.85 & -32.78 & -34.15 \\
\hline & Max. & 237.95 & 202.80 & 33.00 & 20.29 \\
\hline No. of & Positive & 13 & 14 & 2 & 3 \\
\hline & Negative & 18 & 19 & 19 & 12 \\
\hline & Total crosses & 31 & 33 & 21 & 15 \\
\hline \multicolumn{2}{|l|}{ S. E. \pm} & 8.83 & 0.41 & 0.20 & 0.03 \\
\hline
\end{tabular}


Table 1.3

\begin{tabular}{|c|c|c|}
\hline Sr. No. & Hybrids & $\begin{array}{c}\text { Number of } \\
\text { locules per fruit }\end{array}$ \\
\hline & (1) & (2) \\
\hline 1 & ATL-17-02 $\times$ ATL-17-06 & -9.80 \\
\hline 2 & ATL-17-02 $\times$ ATL-17-05 & $-14.29 * *$ \\
\hline 3 & ATL-17-02 × ATL-16-07 & $-42.97 * *$ \\
\hline 4 & ATL-17-02 × GAT-5 & $17.78^{* * *}$ \\
\hline 5 & ATL-17-02 $\times$ DVRT-2 & $-28.99 * *$ \\
\hline 6 & ATL-17-02 $\times 2014 /$ TOLCVRES-1 & $-15.49 * *$ \\
\hline 7 & ATL-17-02 × 2016/TODVAR-2 & 2.00 \\
\hline 8 & ATL-17-02 × 2016/TODVAR-3 & $-16.67 * *$ \\
\hline 9 & ATL-17-02 × 2014/TOLCVRES-3 & $42.22 * *$ \\
\hline 10 & ATL-17-06 $\times$ ATL-17-05 & -5.88 \\
\hline 11 & ATL-17-06 × ATL-16-07 & $-40.58 * *$ \\
\hline 12 & ATL-17-06 × GAT-5 & -3.92 \\
\hline 13 & ATL-17-06 × DVRT-2 & $-31.88 * *$ \\
\hline 14 & ATL-17-06 $\times$ 2014/TOLCVRES-1 & $-40.85 * *$ \\
\hline 15 & ATL-17-06 × 2016/TODVAR-2 & $13.73 * *$ \\
\hline 16 & ATL-17-06 $\times 2016 /$ TODVAR-3 & -5.56 \\
\hline 17 & ATL-17-06 × 2014/TOLCVRES-3 & -13.73 \\
\hline 18 & ATL-17-05 × ATL-16-07 & $-23.19 * *$ \\
\hline 19 & ATL-17-05 × GAT-5 & -8.16 \\
\hline 20 & ATL-17-05 × DVRT-2 & $-28.99 * *$ \\
\hline 21 & ATL-17-05 × 2014/TOLCVRES-1 & $-45.07 * *$ \\
\hline 22 & ATL-17-05 × 2016/TODVAR-2 & $-24.00 * *$ \\
\hline 23 & ATL-17-05 × 2016/TODVAR-3 & $-27.78 * *$ \\
\hline 24 & ATL-17-05 × 2014/TOLCVRES-3 & 2.04 \\
\hline 25 & ATL-16-07 × GAT-5 & -28.99 \\
\hline 26 & ATL-16-07 × DVRT-2 & $-24.64 * *$ \\
\hline 27 & ATL-16-07 × 2014/TOLCVRES-1 & $-26.76 * *$ \\
\hline 28 & ATL-16-07 × 2016/TODVAR-2 & -31.88 \\
\hline 29. & ATL-16-07 × 2016/TODVAR-3 & -28.99 \\
\hline 30 & ATL-16-07 × 2014/TOLCVRES-3 & -33.33 \\
\hline 31 & GAT-5 $\times$ DVRT-2 & -7.25 \\
\hline 32 & GAT-5 $\times 2014 /$ TOLCVRES-1 & $-19.72 * *$ \\
\hline 33 & GAT-5 × 2016/TODVAR-2 & $38.00^{* *}$ \\
\hline 34 & GAT-5 × 2016/TODVAR-3 & $-14.81 * *$ \\
\hline 35 & GAT-5 × 2014/TOLCVRES-3 & 8.89 \\
\hline 36 & DVRT-2 $\times$ 2014/TOLCVRES-1 & $-15.94 * *$ \\
\hline 37 & DVRT-2 × 2016/TODVAR-2 & $14.00^{* * *}$ \\
\hline 38 & DVRT-2 $\times 2016 /$ TODVAR-3 & 9.26 \\
\hline
\end{tabular}




\begin{tabular}{|c|c|c|}
\hline 39 & DVRT-2 $\times 2014 /$ TOLCVRES-3 & $22.22 * *$ \\
\hline 40 & 2014/TOLCVRES-1 × 2016/TODVAR-2 & -36.62 \\
\hline 41 & 2014/TOLCVRES-1 × 2016/TODVAR-3 & -35.21 \\
\hline 42 & 2014/TOLCVRES-1 × 2014/TOLCVRES-3 & -36.62 \\
\hline 43 & 2016/TODVAR-2 × 2016/TODVAR-3 & $7.41 * *$ \\
\hline 44 & 2016/TODVAR-2 $\times$ 2014/TOLCVRES-3 & $78.00 * *$ \\
\hline 45 & 2016/TODVAR-3 × 2014/TOLCVRES-3 & $57.41 * *$ \\
\hline \multirow[t]{2}{*}{ Range } & Min. & -45.07 \\
\hline & Max. & 78.00 \\
\hline \multirow{3}{*}{$\begin{array}{l}\text { No. of } \\
\text { signific } \\
\text { ant } \\
\text { crosses }\end{array}$} & Positive & 18 \\
\hline & Negative & 9 \\
\hline & Total crosses & 27 \\
\hline S. E. \pm & 0.18 & \\
\hline
\end{tabular}

Assessing the heterotic effect over better parent in the crosses derived from genetically diverse parent, in terms of yield, its various attributing traits had been one of the objectives of the present study. It may be positive or negative in direction. Heterobeltiosis in negative direction is generally preferred for the characters like days to initiation of flowering and days to first fruit ripening in tomato. So, for days to initiation of flowering and for days to first fruit ripeningATL-17-02 $\times$ ATL-16-07 and 2016/TODVAR-2 × 2014/TOLCVRES-3 respectively, recorded highest heterobeltiosis.

Then coming to positive heterobeltiosis which is desirable for characters like plant height, average fruit weight, number of fruits per plant, fruit yield per plant, etc., So, for plant height (2016/TODVAR-2 × 2014/ TOLCVRES-3), average fruit weight (ATL16-07 $\times$ GAT-5), number of fruits per plant (GAT-5 × DVRT-2), fruit yield per plant (hybrids ATL-17-05 × 2016/TODVAR-3), 1000 seed weight (ATL-17-02 $\times$ ATL-17-05), pericarp thickness (GAT-5 × 2014/ TOLCVRES-3), number of locules per fruit (2016/TODVAR-2 × 2014/TOLCVRES-3) these crosses are recorded with highest heterobeltiosis.

The results indicated that the crosses exhibited high heterobeltotic effect for yield and its important attributes, might possibly be useful in heterosis breeding programmes for further improvement. These crosses with high heterobeltotic effect also gave an idea to consider these heterotic effects for the production of superior lines.

\section{References}

Ahmad, M., Gul, Z., Khan, Z. U., Iqbal, M., Khan, B., Saleem, M., and Ullah, I. (2015). Study of heterosis in different cross combinations of tomato for yield and yield components. International Journal of Biosciences, 7(2), 12-18.

Aisyah, S. I., Wahyuni, S., Syukur, M., and Witono, J. R. (2016). The estimation of combining ability and heterosis effect for yield and yield components in tomato (Solanum lycopersicum Mill.) at lowland. Ekin Journal of Crop Breeding and Genetics, 2(1), 23-29.

Anonymous (2018b). National Horticulture 
Board, Retrieved from. http://nhb.gov.in/statistics/State_Level/2 017-18-(Final).pdf

Baishya, K. C., Syamal, M. M., and Singh, K. P. (2001). Heterosis studies in tomato (Lycopersicon esculentum Mill.). Journal of Vegetation Science, 28(2), 168-169.

Bhalala, K. C. (2018). Exploitation of heterosis and assessment of combining ability using line $\times$ tester analysis over environments in tomato (Solanum lycopersicum L.) Ph. D., Thesis, Anand Agricultural University, Anand, Gujarat. Retrieved from http://krishikosh.egranth.ac.in/handle/1/ 5810093036

Chauhan, V. B. S., Behera, T. K., and Yadav, R. K. (2014). Studies on Heterosis for yield and its attributing traits in tomato (Solanum lycopersicum L.). International Journal of Agriculture, Environment and Biotechnology,7(1), 95.

Dagade, S. B., Barad, A. V., and Dhaduk, L. K. (2015). Studies on hybrid vigour in $F_{1}$ and its retention in $F_{2}$ for fruit firmness and related traits in tomato. International Journal of Applied Biology and Pharmaceutical Technology, 6(1), 193-199.

Duhan, D., Partap, P. S., Rana, M. K., and Dahiya, M. S. (2005). Heterosis study for quality characters in a line $\times$ tester set of tomato. Haryana Journal of Horticulture Sciences, 34(3-4), 371375.

Fonseca, S., and Patterson, F. L. (1968). Hybrid vigour in a seven parent diallel cross in common wheat (Triticum aestivum L.). Crop Science, 8(5), 85-88.

Hamid, M., Saeid, M., Morteza. A., and Younes, M. (2011). Total soluble solids, titratable acidity and ripening index of tomato in various storage Conditions. Australian Journal of Basic and Applied
Sciences, 5(12), 1723-1726,

Hannan, M. M., Ahmed, M. B., Razvy, M. A., Karim, R., Khatun, M., Haydar, A., Hossain, M., and Roy, U. K. (2007a). Heterosis and correlation of yield and yield components in tomato (Lycopersicon esculentum Mill.). American-Eurasian Journal of Scientific Research, 2(2), 146-150.

Hayman, B.I. (1957). Interaction, heterosis and diallel cross. Genetics, 42, 336355.

Joshi, A., and Thakur, M. C. (2003). Exploitation of heterosis for yield contributing traits in tomato (Lycopersicon esculentum Mill.). Progressive Horticulture, 35(1), 64-68.

Joshi, A., Thakur, M. C., and Kohli, U. K. (2005). Heterosis and combining ability for shelf life, whole fruit firmness and related traits in tomato. Indian Journal of Horticulture, 62(1), 33-36.

Kalloo, G. (1988). Distant hybridization in vegetable crops. Vegetable Breeding, Vol. I, CRC Press, Inc, Fla, USA, pp $137-170$.

Kumar, C. (2016). Heterosis and Combining Ability Studies in Diallel Crosses of Tomato (Solanum lycopersicum L.) (Doctoral dissertation, Institute of Agricultural Sciences, Banaras Hindu University). Retrieved from http://krishikosh.egranth.ac.in/handle/1/ 5810102890

Kumar, P., and Paliwal, A. (2016). Heterosis breeding for quality improvement in tomato (Lycopersicon esculentum Mill.) for cultivation in mid hills of Uttarakhand. International Journal of New Technology and Research, 2(10), 75-78.

Kumari, S., and Sharma, M. K. (2011). Exploitation of heterosis for yield and its contributing traits in tomato (Solanum lycopersicum L.). International Journal of Farm Science, 
$1(2), 45-55$.

Narasimhamurthy, Y. K., and Gowda, P. H. R. (2013). Line $x$ tester analysis in tomato (Solanum lycopersicum L.): Identification of superior parents for fruit quality and yield attributing traits. International Journal of Plant Breeding, 7(1), 50-54.

Panchal B. B., Gurjar, T. D., Kalaria, V. D., and Patel N. B. (2017b). Study of heterosis breeding for processing characters in tomato (Solanum lycopersicum L.). International Journal of Pure and Applied Bioscience, 5(4), 592-600.

Patwary, M. A., Rahman, M. M., Ahmad, S., Miah, M. K., and Barua, H. (2013). Study of heterosis in heat tolerant tomato (Solanum lycopersicum) during summer. Bangladesh Journal of Agricultural Research, 38(3), 531-544.

Rajan, R. E. B. (2014). Heterosis for some quality traits in tomato (Lycopersicon esculentum Mill). Plant Archives, 14(1), 101-104.

Sharma, P., and Bhardwaj, N. (2016). Genetic study of heterosis for yield and quality components in tomato (Solanum lycopersicum L.). Environment and Ecology, 34(3), 832-837.

Singh, J., and Singh, S. (2003). Estimation of heterosis using male sterile lines in tomato (Lycopersicon esculentum). Journal of Vegetation Science, 30(2),
161-163.

Singh, N. B., Wani, S. H., Haribhusan, A., and Nonghtombam, R. (2012). Heterosis studies for yield and its components in tomato (Solanum lycopersicum L.) under valley conditions of Manipur. Vegetos-An International Journal of Plant Research, 25(2), 257-265.

Singh, S. (2005). Genetic analysis of fruit yield and quality characters in tomato (M. Sc. Thesis, Anand Agricultural University, Anand, Gujarat). Retrieved from http://krishikosh.egranth.ac.in/handle/1/ 5810033055

Tiwari, A., and Lal, G. (2004). Studies on heterosis for quantitative and qualitative characters in tomato. Progressive Horticulture, 36(1), 122-127.

Triveni, D., Saidaiah, P., Reddy, K. R., and Pandravada, S. R. (2017). Studies on heterosis for yield and yield contributing traits in tomato (Solanum lycopersicum L.). International Journal of Pure and Applied Bioscience, 5(4), 1677-1685.

Yadav, S. K., Singh, B. K., Baranwal, D. K., and Solankey, S. S. (2013). Genetic study of heterosis for yield and quality components in tomato (Solanum lycopersicum). African Journal of Agricultural Research, 8(44), 55855591.

\section{How to cite this article:}

Mayur Kumar Sonagara, Jyotindra N. Patel, R. R. Acharya and Deepak D. Sharma. 2020. Exploitation of Heterobeltiosis in Tomato (Solanum lycopersicum L.). Int.J.Curr.Microbiol.App.Sci. 9(04): 674-685. doi: https://doi.org/10.20546/ijcmas.2020.904.082 